\title{
MERADGEN 1.0: Monte Carlo generator for the simulation of radiative events in parity conserving doubly-polarized Møller scattering
}

\author{
Andrei Afanasev ${ }^{\mathrm{a}}$ Eugene Chudakov ${ }^{\mathrm{a}}$ Alexander Ilyichev ${ }^{\mathrm{b}}$ \\ Vladimir Zykunov ${ }^{\mathrm{c}}$ \\ a Jefferson Lab, Newport News, VA 23606, USA \\ ${ }^{\mathrm{b}}$ National Scientific and Educational Centre of Particle and High Energy Physics \\ of the Belarusian State University, Minsk, 220040 Belarus \\ ${ }^{\mathrm{c}}$ Joint Institute for Nuclear Research, Dubna, 141980 Russia and Gomel State \\ Technical University, Gomel, 246746 Belarus
}

\begin{abstract}
The Monte Carlo generator MERADGEN 1.0 for the simulation of radiative events in parity conserving doubly-polarized Møller scattering has been developed. Analytical integration wherever it is possible provides rather fast and accurate generation. Some numerical tests and histograms are presented.
\end{abstract}

\section{PROGRAM SUMMARY}

Manuscript title: MERADGEN 1.0: Monte Carlo generator for the simulation of radiative events in parity conserving doubly-polarized Møller scattering Authors: A. Afanasev, E. Chudakov, A. Ilyichev, V. Zykunov

Program title: MERADGEN 1.0

Licensing provisions: none

Programming language: FORTRAN 77

Computer(s) for which the program has been designed: all

Operating system(s) for which the program has been designed: Linux

$R A M$ required to execute with typical data: $1 \mathrm{MB}$

Has the code been vectorised or parallelized?: no

Number of processors used: 1

Email addresses: ily@hep.by (Alexander Ilyichev), zykunov@sunse.jinr.ru (Vladimir Zykunov).

Preprint submitted to Elsevier Science 
Supplementary material: none

Keywords: radiative corrections, Monte Carlo method, Møller Scattering

$\underline{P A C S}$ : 07.05.Tp, 13.40.Ks, 13.88.+e, 25.30.Bf

CPC Library Classification:

External routines/libraries used: none

CPC Program Library subprograms used: none

Nature of problem: simulation of radiative events in parity conserving doublypolarized Møller scattering.

Solution method: Monte Carlo method for simulation within QED, analytical integration wherever it is possible that provides rather fast and accurate generation

Restrictions: none

Unusual features: none

Additional comments: none

Running time: the simulation of $10^{8}$ radiative events for itest $:=1$ takes up to 45 seconds on AMD Athlon 2.80 GHz processor.

\section{Introduction}

The precise measurements in polarized Møller scattering play a very important role in the modern polarimetry. The coincidence detection of the final electron pairs allows to essentially reduce a background of radiative effects [1] that accompany any charge particle scattering, including investigated reaction. However due to finite detector resolution it is impossible to remove all radiative event contributions out of the data. Moreover the additional virtual particle contributions can not be removed by any experimental cuts. Therefore to reach the appropriate accuracy we need to perform the radiative correction procedure consisting in the calculation of these effects within QED and estimate them numerically.

The calculation of the lowest order QED radiative corrections $(\mathrm{RC})$ to polarized Møller scattering was already performed in [2,3] (see also the references therein). In [2] these corrections were calculated exactly (without the ultrarelativistic approximation) but unfortunately also without any experimental cuts. In [3] the similar corrections were performed for longitudinally polarized Møller scattering within the ultrarelativistic approximation. The numerical analysis which is presented in [3] shows that RC to Møller scattering are very sensitive to the missing mass (inelasticity) cuts.

However, the consideration only the missing mass cuts during RC procedure 
is insufficient. The realistic situation corresponds to taking into account the detector geometry that can essentially complicate the integration over the real photon phase space. In such situation an approach based on the Monte Carlo simulation of radiative events is the most adequate. The Monte Carlo generators RADGEN [4] and ELRADGEN [5] for simulation of radiative events in deep inelastic and elastic lepton-nucleon scattering can serve as an examples of such approach.

In present paper a new Monte Carlo generator MERADGEN 1.01 for the simulation of the radiative events in parity conserving part of polarized Møller scattering is presented that can be used for the polarimetry purpose of present and future experiments, for example, in JLab and SLAC. Naturally, as we are taking into consideration the parity conserving effects and the beam energy is rather small (from 1 to $45 \mathrm{GeV}$ ), the weak contributions $(Z$-boson exchange and so one) to this process are negligible. So, we restricted our calculation within QED theory only.

The present paper is organized as following. In Section 2 the kinematics of the investigated process and the generation method are presented. The different contributions to the cross section that is responsible for real photon emission are considered in Section 3. Then in Section 4 the brief structure of the code is discussed, in Section 5 we described the input-output data. In Section 6 we explain how to run tests of our code, some conclusions are given in Section 7. Finally, in Appendices the 4-momenta reconstruction formulas, some lengthy formulas for RC and test output are presented.

\section{Kinematics and Method of Generation}

The lowest order contribution to the observable cross section of Møller scattering reaction

$$
e\left(k_{1}, \xi_{L}\right)+e\left(p_{1}, \eta_{L}\right) \rightarrow e\left(k_{2}\right)+e\left(p_{2}\right)
$$

(in parentheses the 4-momenta and polarization vectors of electrons are presented, and $k_{1}^{2}=k_{2}^{2}=p_{1}^{2}=p_{2}^{2}=m^{2}$ ) can be described by the standard set of Mandelstam variables:

$$
\begin{aligned}
& s=\left(k_{1}+p_{1}\right)^{2}, t=\left(k_{1}-k_{2}\right)^{2}, u=\left(k_{2}-p_{1}\right)^{2}, \\
& s+t+u=4 m^{2}
\end{aligned}
$$

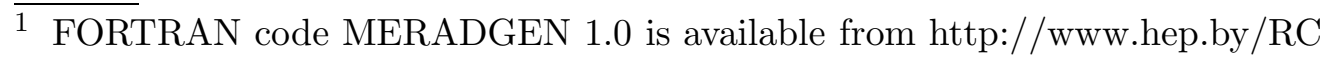


while the beam $\left(\xi_{L}\right)$ and target $\left(\eta_{L}\right)$ polarization vectors read:

$$
\begin{aligned}
\xi_{L} & =\frac{1}{\sqrt{\lambda_{s}}}\left(\frac{s-2 m^{2}}{m} k_{1}-2 m p_{1}\right) \\
\eta_{L} & =\frac{1}{\sqrt{\lambda_{s}}}\left(2 m k_{1}-\frac{s-2 m^{2}}{m} p_{1}\right) .
\end{aligned}
$$

where $\lambda_{s}=s\left(s-4 m^{2}\right)$.

For the definition of the lowest order contribution to Møller scattering it is enough to define an initial beam energy $k_{10}=E_{b}^{\text {Lab }}$ (in Lab. system), a scattering angle $\theta_{\mathrm{CM}}$ (in CM system) of the detected electron with the 4-momentum $k_{2}$ and an azimuthal angle $\phi$. The cross section does not depend on $\phi$ up to taking into account the detector geometry. Let us notice that $E_{b}^{\mathrm{Lab}}$ and $\cos \theta_{\mathrm{CM}}$ can be expressed via the invariants in the following way:

$$
\cos \theta_{\mathrm{CM}}=1+2 t / s=1-2 y, E_{b}^{\mathrm{Lab}}=\frac{s-2 m^{2}}{2 m},
$$

where $y=-t / s$. Therefore instead of the scattering angle and the beam energy for the definition of the Born cross section we can use $s$ and $t$ variables.

For the radiative process with the real photon emission

$$
e\left(k_{1}, \xi_{L}\right)+e\left(p_{1}, \eta_{L}\right) \rightarrow e\left(k_{2}\right)+e\left(p_{2}\right)+\gamma(k)
$$

$\left(k^{2}=0\right)$ three new kinematic variables have to be defined. At first, following notations of ref. [3] we introduce an inelasticity $v=\Lambda^{2}-m^{2}$, where $\Lambda=$ $k_{1}-k_{2}+p_{1}$ and $\Lambda^{2}$ is so-called missing mass square. Maximum value of the inelasticity

$$
v_{\max }=\frac{s t+\sqrt{s\left(s-4 m^{2}\right) t\left(t-4 m^{2}\right)}}{2 m^{2}} \sim s+t
$$

can be defined from the kinematical restriction (see, for example, the ChewLow diagram in [6]). This variable can be directly reconstructed from the data. To remove the contribution of the hard photon emission the events with $v \leq v_{\text {cut }}$ are taking into account, where the value $v_{\text {cut }}$ is far less than $v_{\text {max }}$. The second variable is defined by $t_{1}=\left(p_{2}-p_{1}\right)^{2}=\left(k_{1}-k_{2}-k\right)^{2}$. At last, the third variable should be choose as $z=2 k_{2} k$. Notice that for the radiative process

$$
\cos \theta_{\mathrm{CM}}=1+2 t /(s-v)
$$


To reconstruct the 4-momenta of all particles for radiative process in any system it is enough to determine of variables $s, t, v, t_{1}, z$ and the azimuthal angle $\phi$. As an example, in Appendix A the 4-momenta of electrons and real photon are expressed through these variables and presented in the center of the initial electron mass system.

The simulation of the radiative events can be performed by the following algorithm:

- For the fixed initial energy and $t$ the non-radiative and radiative parts of the observable cross section are calculated.

- The channel of scattering is simulated for the given event in accordance with partial contributions of these two (non-radiative and radiative) positive parts into the observable cross section.

- The angle $\phi$ is simulated uniformly from 0 to $2 \pi$.

- For the radiative event the kinematic variables $v, t_{1}$ and $z$ are simulated in accordance with their calculated distributions.

- The 4-momenta of all final particles in the required system are calculated.

- If the initial $t$ has not a fixed value (i.e. simulated according to the Born probability distribution) then the cross sections have to be stored for reweighing. The $t$-distribution is simulated over the Born cross section, and realistic observed $t$-distribution is calculated as sum of weights, they are ratios of the observable and Born cross sections.

Let us consider some important steps of simulation of the radiative events in more details.

\section{Non-radiative and radiative parts of the observable cross section}

Here we consider the observable cross section that has a form 2 :

$$
\sigma^{o b s}\left(v_{c u t}\right)=\sigma^{0}+\sigma^{R V}\left(v_{c u t}\right)+\sigma_{F}^{R}\left(v_{c u t}\right),
$$

where $\sigma^{0}$ is the Born contribution, $\sigma^{R V}$ is an infrared divergency free sum of the contributions of the additional virtual particles and the "infrared" part of the real photon emission, $\sigma_{F}^{R}$ is the infrared divergency free part of the unobservable photon emission. The explicit expressions for each term of equation (8) can be found in [3]. Since the expressions for the two virtual photon contribution as well as $\delta_{1}^{H}$ and $\delta_{1}^{S}$ in [3] contain the misprints (fortunately, it is not seriously reflecting on the numerical estimations presented in [3]),

$\overline{2}$ Here and later we consider the differential cross section $\sigma \equiv d \sigma / d y$ 
the additional virtual particle contributions together with $\delta_{1}^{H}$ and $\delta_{1}^{S}$ are also presented in Appendix B.

Now we consider $\sigma_{F}^{R}$ in more detail: before integration over the inelasticity and the real photon phase space it can be presented as

$$
\sigma_{F}^{R}\left(v_{c u t}\right)=\frac{\alpha^{3}}{4 s} \int_{0}^{v_{c u t}} d v \int d \Gamma_{k}\left(\left|M_{R}\right|^{2}-4 F^{I R}\left|M_{0}\right|^{2}\right)
$$

where $M_{R}$ is a sum of matrix elements contributed to the real photon emission (see Appendix $\mathrm{C}$ for details), while $M_{0}$ is a Born matrix element. The real photon phase space reads

$$
d \Gamma_{k}=\frac{1}{\pi} \frac{d^{3} k}{k_{0}} \delta\left((\Lambda-k)^{2}-m^{2}\right)=\frac{1}{4 \pi} \frac{d t_{1} d z}{\sqrt{-\Delta\left(k_{1}, k_{2}, p_{1}, k\right)}}
$$

where $\Delta\left(k_{1}, k_{2}, p_{1}, k\right)$ is the Gram determinant. At last,

$$
\begin{aligned}
-F^{I R}= & \frac{m^{2}}{z^{2}}+\frac{m^{2}}{z_{1}^{2}}+\frac{m^{2}}{v^{2}}+\frac{m^{2}}{v_{1}^{2}}+\frac{s-2 m^{2}}{z_{1} v_{1}}+\frac{s-2 m^{2}}{z v}+ \\
& +\frac{t-2 m^{2}}{z_{1} z}+\frac{t-2 m^{2}}{v_{1} v}+\frac{u_{0}-2 m^{2}}{z_{1} v}+\frac{u_{0}-2 m^{2}}{v_{1} z}
\end{aligned}
$$

where $z_{1}=2 k k_{1}, v_{1}=2 k p_{1}$ and $u_{0}=s+t-4 m^{2}$.

For separation of the cross section (8) into the radiative and non-radiative parts it is necessary to introduce a new fictitious parameter $v_{\min }$ associated with missing mass square resolution. Then the equation (9) can be rewrite in the following way

$$
\begin{aligned}
\sigma_{F}^{R}\left(v_{\text {cut }}\right)= & \sigma^{r}\left(v_{\text {cut }}, v_{\text {min }}\right)+\frac{\alpha^{3}}{4 s} \int_{0}^{v_{\text {min }}} d v \int d \Gamma_{k}\left(\left|M_{R}\right|^{2}-4 F^{I R}\left|M_{0}\right|^{2}\right)- \\
& -\frac{\alpha^{3}}{s} \int_{v_{\text {min }}}^{v_{c u t}} d v \int d \Gamma_{k} F^{I R}\left|M_{0}\right|^{2}
\end{aligned}
$$

where

$$
\sigma^{r}\left(v_{c u t}, v_{\text {min }}\right)=\frac{\alpha^{3}}{16 \pi s} \int_{v_{\text {min }}}^{v_{c u t}} d v \int_{t_{1}^{\text {min }}}^{t_{1}^{\max }} d t_{1} \int_{z_{\text {min }}}^{z^{\max }} \frac{d z}{\sqrt{-\Delta\left(k_{1}, k_{2}, p_{1}, k\right)}}\left|M_{R}\right|^{2}=
$$




$$
=\int_{v_{\text {min }}}^{v_{c u t}} d v \int_{t_{1}^{\text {min }}}^{t_{1}^{\max }} d t_{1} \int_{z_{\text {min }}}^{z_{\max }} d z \frac{d^{3} \sigma^{r}\left(v_{c u t}, v_{\text {min }}\right)}{d v d t_{1} d z}
$$

is the radiative part of the cross section. The limits of the integration over $z$ are defined from the equation $\Delta\left(k_{1}, k_{2}, p_{1}, k\right)=0$, while $t_{1}^{\min }$ and $t_{1}^{\max }$ can be found from $z^{\min }=z^{\max }$ (see [6] for details).

Since the observable cross section (8) is the sum of the radiative and nonradiative parts

$$
\sigma^{o b s}\left(v_{c u t}\right)=\sigma^{r}\left(v_{c u t}, v_{\text {min }}\right)+\sigma^{n r}\left(v_{c u t}, v_{\text {min }}\right),
$$

we immediately find that

$$
\begin{aligned}
\sigma^{n r}\left(v_{c u t}, v_{\text {min }}\right)= & \sigma^{0}+\sigma^{R V}\left(v_{c u t}\right)+\frac{\alpha^{3}}{4 s} \int_{0}^{v_{\min }} d v \int d \Gamma_{k}\left(\left|M_{R}\right|^{2}-4 F^{I R}\left|M_{0}\right|^{2}\right)- \\
& -\frac{\alpha^{3}}{s} \int_{v_{\text {min }}}^{v_{c u t}} d v \int d \Gamma_{k} F^{I R}\left|M_{0}\right|^{2}
\end{aligned}
$$

Let us notice that the explicit formulae both for $d^{3} \sigma^{r}\left(v_{c u t}, v_{\min }\right) / d v d t_{1} d z$ in the equation (13) and $\sigma^{n r}\left(v_{c u t}, v_{\text {min }}\right)$ allow us to start the generation of the radiative events. However to speed up the process of generation it is useful to perform the integration over $z$ and $t_{1}$ analytically. So, the following analytical expressions

$$
\begin{aligned}
\frac{d \sigma^{r}\left(v_{c u t}, v_{\text {min }}\right)}{d v} & =\int_{t_{1}^{\text {min }}}^{t_{1}^{\max }} d t_{1} \int_{z^{\text {min }}}^{z^{\max }} d z \frac{d^{3} \sigma^{r}\left(v_{c u t}, v_{\text {min }}\right)}{d v d t_{1} d z} \\
\frac{d^{2} \sigma^{r}\left(v_{c u t}, v_{\text {min }}\right)}{d v d t_{1}} & =\int_{z^{\text {min }}}^{z_{\text {max }}} d z \frac{d^{3} \sigma^{r}\left(v_{c u t}, v_{\text {min }}\right)}{d v d t_{1} d z}
\end{aligned}
$$

are incorporated in our Monte Carlo program. The analytical integration can be performed in a standard way (see, for example, $[2,6]$ and references therein).

At the end of this section it should be noted that according to the equation (14) the non-radiative and radiative contributions to the cross section depend on $v_{c u t}$ and $v_{\text {min }}$, while the observable cross section depends on $v_{c u t}$ only. 


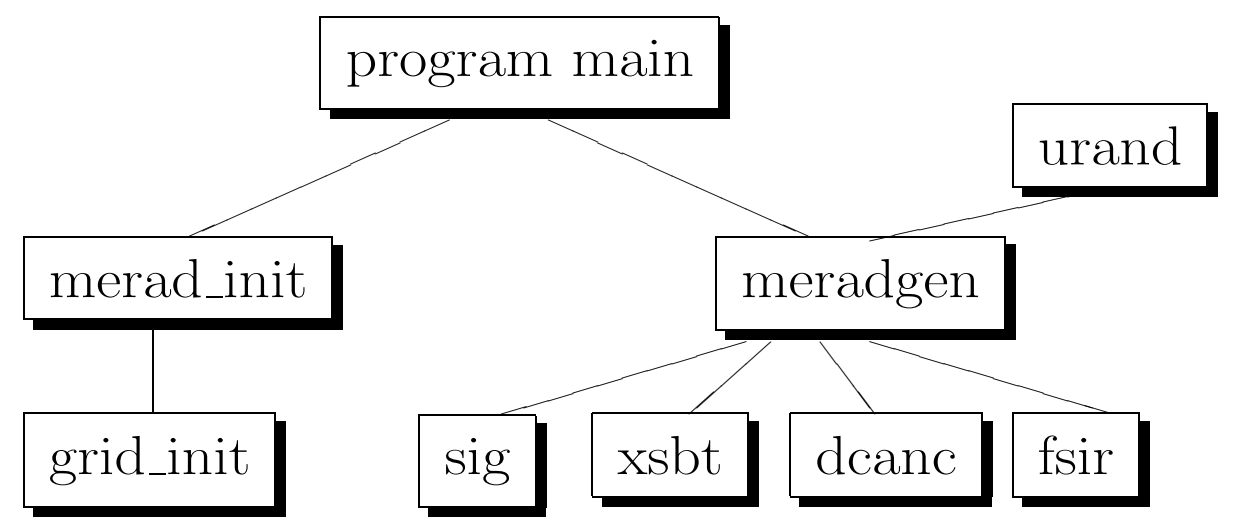

Fig. 1. The structure of the program MERADGEN 1.0

\section{The structure of the program and radiative event simulation}

The structure of the Monte Carlo generator MERADGEN 1.0 is presented in fig. 1. The main blocks mean:

- merad_init — here we define all constants which are necessary for generation;

- grid_init — here we prepare the grids for generation of kinematic variables, really we approximate the theoretical curve by some sets of segments;

- urand - random number generator (flat);

- sig - the Born cross section and part of the virtual contribution (vertices and self energies);

- xsbt - box contribution;

- dcanc - the contribution with cancellation of the infrared divergency;

- fsir - analytical cross sections $d \sigma^{r}\left(v_{c u t}, v_{\text {min }}\right) / d v, d^{2} \sigma^{r}\left(v_{c u t}, v_{\text {min }}\right) / d v d t_{1}$ and $d^{3} \sigma^{r}\left(v_{c u t}, v_{\text {min }}\right) / d v d t_{1} d z$.

For the convenience sake we split our programm into 4 FORTRAN-files:

- run.f - main program for the event generation;

- test.f - main program for the tests;

- meradgen.f - the collection of main functions and subroutines of MERADGEN 1.0;

- fsir.f - function fsir for calculation of the analytical cross sections that are presented above.

For the events (tests) generation we need to run "make" ("make test") command.

Now let us consider the input-output data in more details. 


\section{Input-output data}

As an input data MERADGEN 1.0 uses 4-momentum of the virtual photon vpgen $:=k_{1}-k_{2}$ that generated in CM system externally, energy of electrons and degree of electrons polarization. There is only one variable itest in MERADGEN 1.0 that responsible for output. If itest $:=0$ the output data are gathered in two common blocks (see file output.inc):

common/variables/vgen,t1gen,zgen,weight,ich

and

common/vectors/vprad,phirad.

Here vgen, t1gen and zgen are generated photonic variables $v, t_{1}$ and $z$ respectively, (they are necessary first of all for the test), weight is a ratio of the observable cross section to the Born one, variable $i c h$ shows the radiative $(i c h:=1)$ or non-radiative $(i c h:=0)$ scattering channel, the 4-momentum vprad $:=p_{2}-p_{1}$ and photonic 4 -momentum phirad $:=k$ also defined in CM system.

Here we have to do some remarks: 1) for non-radiative events vgen $:=0$, t1gen $:=t, z:=0$, vprad $:=$ vpgen and phirad $:=0$, and 2 ) as it was mentioned above, the variable $v$ can be reconstructed experimentally and the events with hard photons $v_{c u t}<v \leq v_{\max }$ usually remove from the data. In order to speed up the process generation, we generated variable $v$ from $v_{\text {min }}$ up to $v_{\text {cut }}=(s+t) / 2 \sim v_{\max } / 2$.

Now let us consider one sample of generation. It is well known that for the elastic process (1) the energy of the detected electron $k_{20}$ can be directly defined via the scattering angle $\theta_{\mathrm{CM}}$ and the initial electron beam energy in the following way:

$$
E_{2}^{\mathrm{Lab}} \approx \cos ^{2} \frac{\theta_{\mathrm{CM}}}{2} E_{b}^{\mathrm{Lab}}
$$

but when we deal with the real photon emission (5) the process becomes inelastic and, as a result, the "elastic" equation (17) is broken and we have the 2-dimensional distribution over $E_{2}^{\mathrm{Lab}}$ and $\cos \theta_{\mathrm{CM}}$. From fig. 2 one can see that this distribution has a sharp peak near an "elastic" line described by the equation (17). 

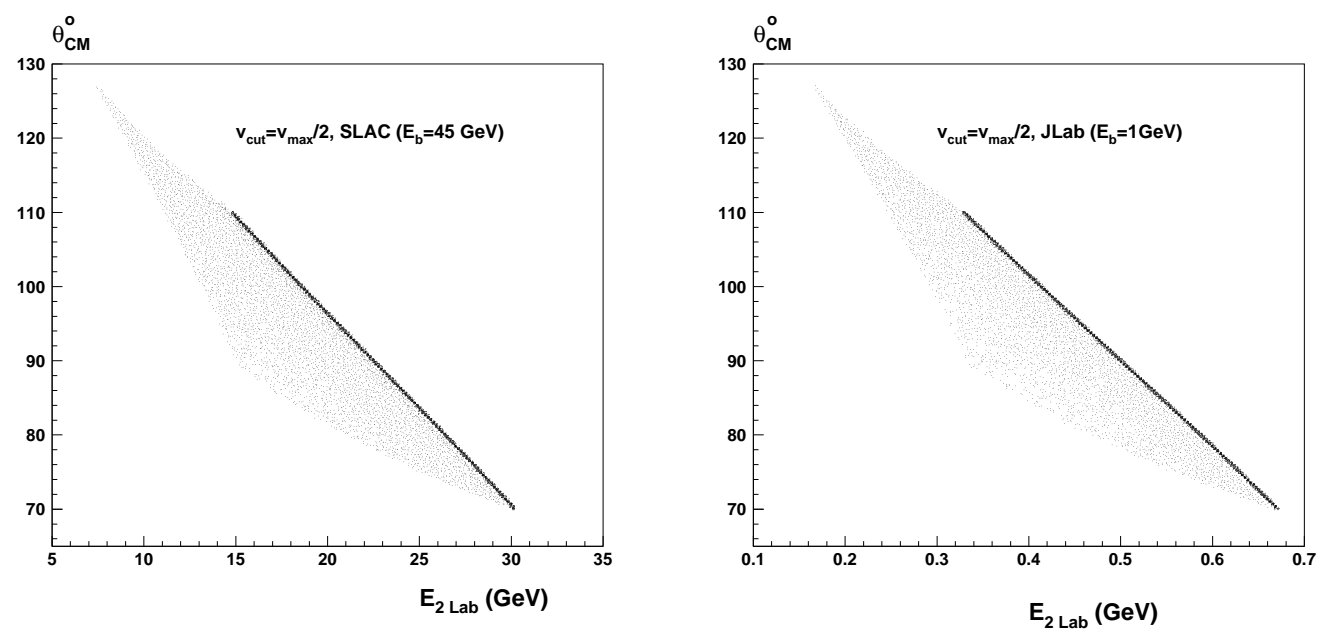

Fig. 2. The 2-dimensional distributions of the scattering electron energy in lab. system and scattering angle $\theta$ in $\mathrm{CM}$ system

\section{Test runs}

For our test runs we use the fact that if the events are simulated correctly their distributions over variables $v, t_{1}$ and $z$ must obey to the corresponding probability distributions:

$$
\begin{array}{rlrl}
\rho(v) & =\frac{1}{N_{v}} \frac{d \sigma^{r}\left(v_{c u t}, v_{\text {min }}\right)}{d v}, & N_{v}=\sigma^{r}\left(v_{c u t}, v_{\text {min }}\right), \\
\rho\left(t_{1}\right)=\frac{1}{N_{t_{1}}} \frac{d^{2} \sigma^{r}\left(v_{c u t}, v_{\text {min }}\right)}{d v d t_{1}}, & N_{t_{1}}=\frac{d \sigma^{r}\left(v_{c u t}, v_{\text {min }}\right)}{d v}, \\
\rho(z)=\frac{1}{N_{z}} \frac{d^{3} \sigma^{r}\left(v_{c u t}, v_{\text {min }}\right)}{d v d t_{1} d z}, & N_{z}=\frac{d^{2} \sigma^{r}\left(v_{c u t}, v_{\text {min }}\right)}{d v d t_{1}} .
\end{array}
$$

Then for generation of $\rho(v), \rho\left(t_{1}\right)$ or $\rho(z)$ distributions one has to put in the file test.f the value of variable itest such as: itest $:=1$, itest $:=2$ or itest $:=3$, respectively, next to type "make test" and, at last, "./test.exe". The value rgen/rcalc, i.e. ratio of generated $\rho$-distribution to corresponding calculated cross section should be near unit. In Appendix $\mathrm{D}$ the test outputs for $v, t_{1}, z$ generation with $P=1$ (see formula (B.2)), $E_{b}^{\mathrm{Lab}}=45 \mathrm{GeV}, \theta_{C M}=90^{0}, 20$ bins for the histogramming and $10^{8}$ radiative events are presented.

The simulated distributions of the photonic variables for the SLAC E158 experiment kinematic conditions and for the different degrees of polarization are presented in fig. 3 (all of parameters are noted there). We suppose $v_{\text {min }}$ rather small: $v_{\min }=2 \times 10^{-2} E_{b}^{\mathrm{Lab}} \mathrm{m}$. In the fig. 3 we can see clearly the divergent behavior of the distributions at $v \rightarrow 0, z \rightarrow z^{\text {min,max }}, t_{1} \rightarrow t_{1}^{\text {min }}$ corresponding to the infrared singularity at the $v_{\min } \rightarrow 0$. Also it can be seen the physical, 

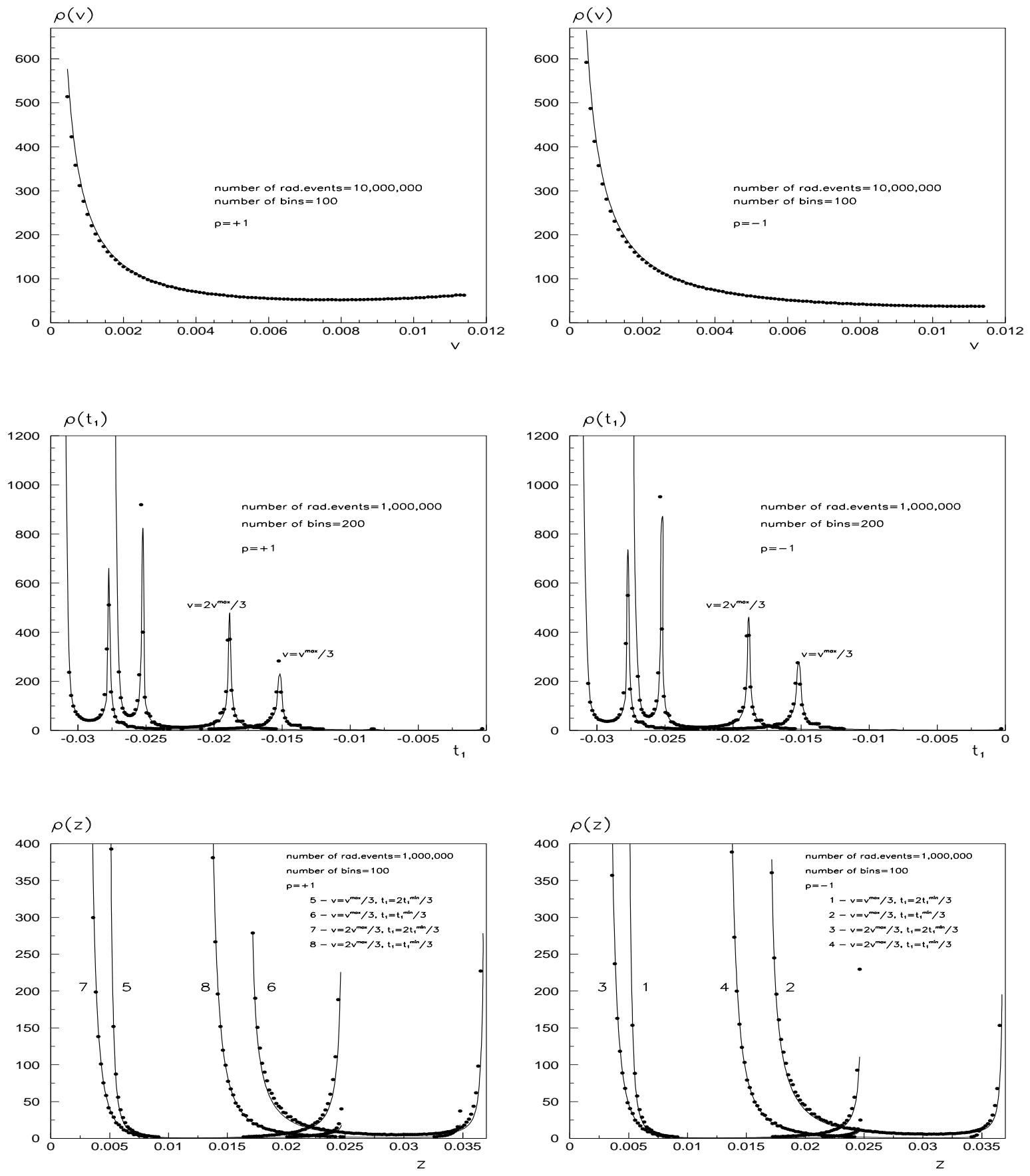

Fig. 3. $v, t_{1}, z$-histograms (points) and corresponding probability densities (curves) for E158 (SLAC) kinematic conditions $\left(E_{b}^{\mathrm{Lab}}=45 \mathrm{GeV}\right)$ 
so-called $z$-and $z_{1}$-peaks of distribution $\rho\left(t_{1}\right)$.

The other test with itest $:=4$ consists in the cross-check of the accuracy of vector reconstruction. So, using all 4-momenta we reproduce the value of generated invariant and compare them with the generated value. In this test we also calculate the photonic mass square as m2gamma := phirad $(4)^{2}-\operatorname{phirad}(1)^{2}-\operatorname{phirad}(2)^{2}-\operatorname{phirad}(3)^{2}$, which should be near zero. In last part of Appendix D we can see good coincidence reconstructed and generated invariants in different (random) kinematical points and very small values of photonic mass square.

\section{Conclusion}

In this paper the Monte Carlo generator MERADGEN 1.0 serving for the simulation of radiative events in parity conserving longitudinally doubly-polarized Møller scattering is presented. Following for the absolute necessity of both accuracy and quickness for our program we have developed the fast and high precise code using analytical integration wherever it was possible. MERADGEN 1.0 can be employed for the radiative corrections procedure in experiments with the complex detector geometry, such as SLAC E158 experiment and experiments of modern polarimetry (JLAB, SLAC).

\section{Acknowledgments}

The authors would like to thank Igor Akushevich, Yury Kolomensky, Nikolai Shumeiko and Juan Suarez for stimulating discussions. V.Z. (A.I.) would like to thank SLAC (JLab) staff for their generous hospitality during their visits.

\section{Appendix A 4-momenta reconstruction}

The 4-momentum definition in the center of mass system of the initial electrons for Møller process with real photon emission is shown in fig. 4 and can be presented in the form:

$$
\begin{aligned}
& k_{1}=\left(k_{10}, 0,0,\left|\vec{k}_{1}\right|\right), p_{1}=\left(p_{10}, 0,0,-\left|\vec{p}_{1}\right|\right), \\
& k_{2}=\left(k_{20}, k_{21}, k_{22}, k_{23}\right), p_{2}=\left(p_{20}, p_{21}, p_{22}, p_{23}\right), \\
& k=\left(k_{0}, k_{1}, k_{2}, k_{3}\right),
\end{aligned}
$$




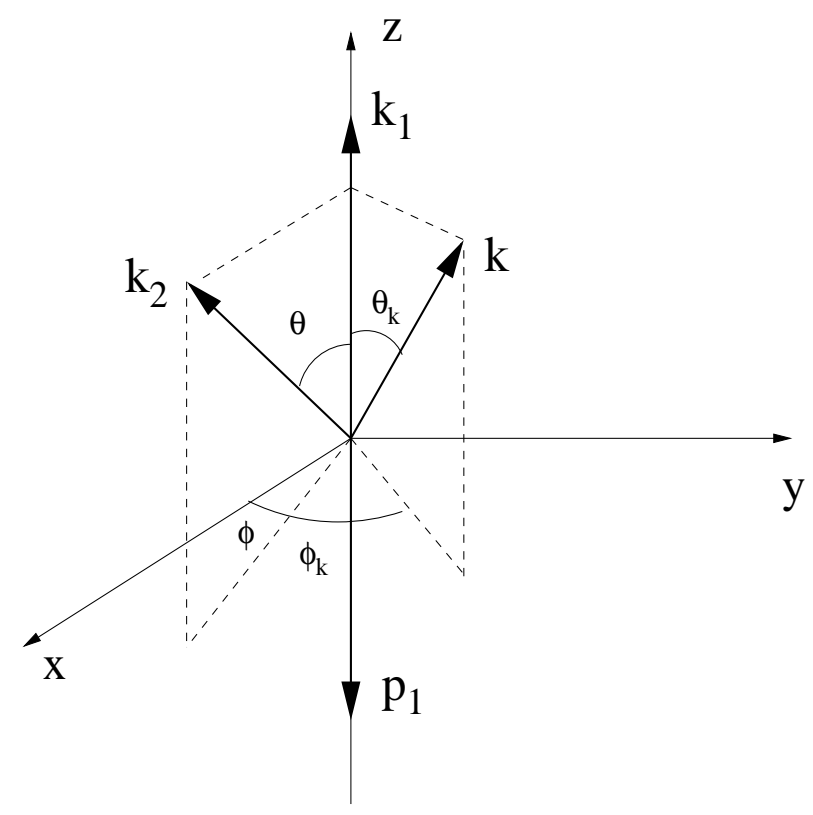

Fig. 4. Definition of momenta and angles in center-of-mass frame

while their components can be expressed via the invariants and azimuthal angle $\phi$ (that is usually generated uniformly) in the following way:

$$
\begin{aligned}
& k_{10}=p_{10}=\frac{1}{2} \sqrt{s},\left|\vec{k}_{1}\right|=\left|\vec{p}_{1}\right|=\frac{\sqrt{\lambda_{s}}}{2 \sqrt{s}}, \\
& k_{20}=\frac{s-v}{2 \sqrt{s}}, k_{21}=\sqrt{\frac{\lambda_{3}}{\lambda_{s}}} \cos \phi, k_{22}=\sqrt{\frac{\lambda_{3}}{\lambda_{s}}} \sin \phi, k_{23}=\frac{\sqrt{s} \lambda_{2}}{2 \sqrt{\lambda_{s}}}, \\
& p_{20}=\frac{s-z}{2 \sqrt{s}}, \\
& p_{21}=-\frac{\sqrt{\lambda_{s} \lambda_{1} \lambda_{8}} \sin \phi+\left(4 \lambda_{3} \lambda_{4}+s \lambda_{2} \lambda_{7}\right) \cos \phi}{4 \lambda_{1} \sqrt{\lambda_{s} \lambda_{3}}}, \\
& p_{22}=\frac{\sqrt{\lambda_{s} \lambda_{1} \lambda_{8}} \cos \phi-\left(4 \lambda_{3} \lambda_{4}+s \lambda_{2} \lambda_{7}\right) \sin \phi}{4 \lambda_{1} \sqrt{\lambda_{s} \lambda_{3}}}, \\
& p_{23}=\frac{\sqrt{s}\left(\lambda_{7}-\lambda_{2} \lambda_{4}\right)}{2 \lambda_{1} \sqrt{\lambda_{s}}}, \\
& k_{0}=\frac{v+z}{2 \sqrt{s}}, \\
& k_{1}=\frac{\sqrt{\lambda_{s} \lambda_{1} \lambda_{8}} \sin \phi+\left(4 \lambda_{3} \lambda_{6}-s \lambda_{2} \lambda_{7}\right) \cos \phi}{4 \lambda_{1} \sqrt{\lambda_{s} \lambda_{3}}} \\
& k_{2}=\frac{-\sqrt{\lambda_{s} \lambda_{1} \lambda_{8}} \cos \phi+\left(4 \lambda_{3} \lambda_{6}-s \lambda_{2} \lambda_{7}\right) \sin \phi}{4 \lambda_{1} \sqrt{\lambda_{s} \lambda_{3}}}
\end{aligned}
$$




$$
k_{3}=\frac{\sqrt{s}\left(\lambda_{7}+\lambda_{2} \lambda_{6}\right)}{2 \lambda_{1} \sqrt{\lambda_{s}}}
$$

Here

$$
\begin{array}{ll}
\lambda_{1}=(s-v)^{2}-4 s m^{2}, & \lambda_{2}=2 t+s-v-4 m^{2}, \\
\lambda_{3}=-s t\left(s+t-v-4 m^{2}\right)-m^{2} v^{2}, & \lambda_{4}=s\left(s-v-4 m^{2}\right)-(s+v) z, \\
\lambda_{5}=v z(s-v-z)-m^{2}(v+z)^{2}, & \lambda_{6}=s(v-z)-v(v+z), \\
\lambda_{7}=\left(s+2 t_{1}-z-4 m^{2}\right) \lambda_{1}-\lambda_{2} \lambda_{4}, & \lambda_{8}=16 \lambda_{3} \lambda_{5}-\lambda_{7}^{2} .
\end{array}
$$

As a result, the angles in fig. 4 can be expressed via the invariants in the following way:

$$
\cos \theta=\frac{s \lambda_{2}}{\sqrt{\lambda_{s} \lambda_{1}}}, \cos \theta_{k}=\frac{s\left(\lambda_{7}+\lambda_{2} \lambda_{6}\right)}{(v+z) \lambda_{1} \sqrt{\lambda_{s}}}, \tan \phi_{k}=\frac{\sqrt{\lambda_{s} \lambda_{1} \lambda_{8}}}{s \lambda_{2} \lambda_{7}-4 \lambda_{3} \lambda_{6}}
$$

\section{Appendix B Additional virtual particle, $\delta_{1}^{H}$ and $\delta_{1}^{S}$ contributions}

The virtual contributions to Møller scattering can be separated into three parts:

$$
\sigma^{\mathrm{V}}=\sigma^{\mathrm{S}}+\sigma^{\mathrm{Ver}}+\sigma^{\mathrm{Box}}
$$

where 1) $\sigma^{\mathrm{S}}$ is a virtual photon self-energy contribution, 2) $\sigma^{\mathrm{Ver}}$ is a vertex function contribution, 3) $\sigma^{\text {Box }}$ is a box contribution. Now we consider each of them.

(1) The contribution of the virtual photon self energies (including the photon vacuum polarization by hadrons) to the cross section looks like

$$
\begin{aligned}
\sigma^{\mathrm{S}}= & \frac{4 \pi \alpha^{2}}{t^{2}} \operatorname{Re}\left(-\frac{1}{t} \hat{\Sigma}_{T}^{\gamma}(t)+\Pi_{h}(-t)\right)\left[(1+P) \frac{u^{2}}{s}-(1-P) \frac{s^{2}}{u}\right] \\
& +(t \leftrightarrow u) .
\end{aligned}
$$

Here $P=P_{B} P_{T}$, where $P_{B}\left(P_{T}\right)$ is the beam (target) polarization, $\hat{\Sigma}_{T}^{\gamma}(-t)$ is the renormalized transverse part of the $\gamma$-self-energy [7] (this part includes vacuum polarization by $e, \mu$ and $\tau$ charged leptons: in corresponding formula of [7] we should take a summing index $f=e, \mu, \tau)$. The hadronic part of the photonic vacuum polarization associated with light 
quarks can be directly obtained from the data on process $e^{+} e^{-} \rightarrow$ hadrons via dispersion relations. Here we use parameterization of [8]

$$
\operatorname{Re} \Pi_{h}(-t) \cong A+B \ln (1+C|t|)
$$

with updated parameters $\mathrm{A}, \mathrm{B}, \mathrm{C}$ in different energy regions.

(2) For the contribution of the electron vertices we used the results of the paper [7] (see also references therein). We can obtain the vertex part as

$$
\sigma^{\mathrm{Ver}}=\frac{2 \alpha^{3}}{t^{2}}\left[(1+P) \frac{u^{2}}{s}-(1-P) \frac{s^{2}}{u}\right] \Lambda_{1}\left(t, m^{2}\right)+(t \leftrightarrow u),
$$

where

$$
\Lambda_{1}\left(t, m^{2}\right)=-2 \ln \frac{|t|}{\lambda^{2}}\left(\ln \frac{|t|}{m^{2}}-1\right)+\ln \frac{|t|}{m^{2}}+\ln ^{2} \frac{|t|}{m^{2}}+\frac{\pi^{2}}{3}-4 .
$$

(3) Recalculated here expressions for the box cross section are slightly different from presented in [3] (we correct the misprints in the expression (16) of $[3])$

$$
\begin{aligned}
\sigma^{\text {Box }}= & \frac{2 \alpha^{3}}{t}\left[\frac{1+P}{s}\left(\frac{2 u^{2}}{t} \ln \frac{s}{|u|} \ln \frac{\sqrt{s|u|}}{\lambda^{2}}-\delta_{(\gamma \gamma)}^{1}\right)\right. \\
& \left.-\frac{1-P}{u}\left(\frac{2 s^{2}}{t} \ln \frac{s}{|u|} \ln \frac{\sqrt{s|u|}}{\lambda^{2}}-\delta_{(\gamma \gamma)}^{2}\right)\right]+(t \leftrightarrow u),
\end{aligned}
$$

The expressions $\delta_{(\gamma \gamma)}^{1,2}$ have the form:

$$
\begin{aligned}
& \delta_{(\gamma \gamma)}^{1}=l_{s}^{2} \frac{s^{2}+u^{2}}{2 t}-l_{s} u-\left(l_{x}^{2}+\pi^{2}\right) \frac{u^{2}}{t}, \\
& \delta_{(\gamma \gamma)}^{2}=l_{s}^{2} \frac{s^{2}}{t}+l_{x} s-\left(l_{x}^{2}+\pi^{2}\right) \frac{s^{2}+u^{2}}{2 t}
\end{aligned}
$$

and logarithms look like

$$
l_{s}=\ln \frac{s}{|t|}, l_{x}=\ln \frac{u}{t}
$$

It should be noted that vertex and box parts contain the infrared divergence through the appearance of the fictitious photon mass $\lambda$. The infrared part from virtual cross section can be extracted in a simple way:

$$
\sigma_{I R}^{V}=\sigma^{V}-\sigma^{V}\left(\lambda^{2} \rightarrow s\right)=-\frac{2 \alpha}{\pi} \ln \frac{s}{\lambda^{2}}\left(\ln \frac{t u}{m^{2} s}-1\right) \sigma^{0}
$$

The correct expressions for $\delta_{1}^{H}$ and $\delta_{1}^{S}$ read 


$$
\begin{aligned}
\delta_{1}^{H}= & -\frac{1}{2} l_{m}^{2}+\left(\ln \frac{t^{2}(s+t)^{2}\left(s-v_{\text {max }}\right)}{s\left(s+t-v_{\max }\right)^{2} v_{\max }\left(v_{\max }-t\right)}+1\right) l_{m}-\frac{1}{2} \ln ^{2} \frac{v_{\text {max }}}{|t|}- \\
& -\ln ^{2}\left(1-\frac{v_{\max }}{t}\right)+\ln \frac{s+t}{s+t-v_{\max }} \ln \frac{(s+t)\left(s+t-v_{\max }\right)}{t^{2}}+ \\
& +\ln \frac{s-v_{\max }}{|t|} \ln \frac{s-v_{\max }}{s}+\ln \frac{v_{\max }}{|t|}+2\left[\operatorname{Li}_{2}\left(\frac{v_{\max }}{s}\right)-\operatorname{Li}_{2}\left(\frac{v_{\max }}{t}\right)-\right. \\
& \left.-\operatorname{Li}_{2}\left(\frac{v_{\max }}{s+t}\right)\right]+\operatorname{Li}_{2}\left(\frac{s-v_{\max }}{s}\right)-\operatorname{Li}_{2}\left(\frac{t-v_{\max }}{t}\right)-\frac{\pi^{2}}{6}, \\
\delta_{1}^{S}= & -\frac{5}{2} l_{m}^{2}+\left(3-2 l_{r}\right) l_{m}-\left(l_{m}-1\right) \ln \frac{s(s+t)}{t^{2}}-\frac{1}{2} l_{r}^{2}-\frac{\pi^{2}}{3}+1 .
\end{aligned}
$$

\section{Appendix C Matrix element of the real photon emission}

The sum of the matrix elements contributed to the real photon emission in Møller process reads:

$$
\begin{aligned}
M_{R}^{\alpha} & =\frac{1}{t_{1}} \bar{u}\left(k_{2}\right) \Gamma_{\mu \alpha}\left(k_{2}, k_{1}\right) u\left(k_{1}\right) \bar{u}\left(p_{2}\right) \gamma_{\mu} u\left(p_{1}\right)+ \\
& +\frac{1}{t} \bar{u}\left(k_{2}\right) \gamma_{\mu} u\left(k_{1}\right) \bar{u}\left(p_{2}\right) \Gamma_{\mu \alpha}\left(p_{2}, p_{1}\right) u\left(p_{1}\right)- \\
& -\frac{1}{u} \bar{u}\left(p_{2}\right) \Gamma_{\mu \alpha}\left(p_{2}, k_{1}\right) u\left(k_{1}\right) \bar{u}\left(k_{2}\right) \gamma_{\mu} u\left(p_{1}\right)- \\
& -\frac{1}{z_{2}} \bar{u}\left(p_{2}\right) \gamma_{\mu} u\left(k_{1}\right) \bar{u}\left(k_{2}\right) \Gamma_{\mu \alpha}\left(k_{2}, p_{1}\right) u\left(p_{1}\right) .
\end{aligned}
$$

The conjugate matrix element can be found in a simple way:

$$
\begin{aligned}
\bar{M}_{R}^{\alpha}= & \frac{1}{t_{1}} \bar{u}\left(k_{1}\right) \bar{\Gamma}_{\nu \alpha}\left(k_{1}, k_{2}\right) u\left(k_{2}\right) \bar{u}\left(p_{1}\right) \gamma_{\nu} u\left(p_{2}\right)+ \\
& +\frac{1}{t} \bar{u}\left(k_{1}\right) \gamma_{\nu} u\left(k_{2}\right) \bar{u}\left(p_{1}\right) \bar{\Gamma}_{\nu \alpha}\left(p_{1}, p_{2}\right) u\left(p_{2}\right)- \\
& -\frac{1}{u} \bar{u}\left(k_{1}\right) \bar{\Gamma}_{\nu \alpha}\left(k_{1}, p_{2}\right) u\left(p_{2}\right) \bar{u}\left(p_{1}\right) \gamma_{\nu} u\left(k_{2}\right)- \\
& -\frac{1}{z_{2}} \bar{u}\left(k_{1}\right) \gamma_{\nu} u\left(p_{2}\right) \bar{u}\left(p_{1}\right) \bar{\Gamma}_{\nu \alpha}\left(p_{1}, k_{2}\right) u\left(k_{2}\right) .
\end{aligned}
$$

Here $z_{2}=z-s-t_{1}+4 m^{2}$ and

$$
\Gamma_{\mu \alpha}(a, b)=\left(\frac{b_{\alpha}}{k b}-\frac{a_{\alpha}}{k a}\right) \gamma_{\mu}-\frac{\gamma_{\mu} \hat{k} \gamma_{\alpha}}{2 b k}-\frac{\gamma_{\alpha} \hat{k} \gamma_{\mu}}{2 a k}
$$




$$
\bar{\Gamma}_{\nu \alpha}(a, b)=-\left(\frac{b_{\alpha}}{k b}-\frac{a_{\alpha}}{k a}\right) \gamma_{\nu}-\frac{\gamma_{\nu} \hat{k} \gamma_{\alpha}}{2 b k}-\frac{\gamma_{\alpha} \hat{k} \gamma_{\nu}}{2 a k}
$$

By introducing

$$
\begin{aligned}
& S\left(a_{1}, a_{2}, a_{3}, a_{4}\right)=\operatorname{Tr}\left[a_{1} \rho\left(a_{2}\right) a_{3} \Lambda\left(a_{4}\right)\right] \\
& S\left(a_{1}, a_{2}, a_{3}, a_{4}, a_{5}, a_{6}, a_{7}, a_{8}\right)=\operatorname{Tr}\left[a_{1} \rho\left(a_{2}\right) a_{3} \Lambda\left(a_{4}\right) a_{5} \rho\left(a_{6}\right) a_{7} \Lambda\left(a_{8}\right)\right],
\end{aligned}
$$

where

$$
\begin{aligned}
& u\left(k_{1}\right) \bar{u}\left(k_{1}\right)=\rho\left(k_{1}\right)=\frac{1}{2}\left(\hat{k_{1}}+m\right)\left(1-P_{B} \gamma_{5} \hat{\xi}_{L}\right), \\
& u\left(p_{1}\right) \bar{u}\left(p_{1}\right)=\rho\left(p_{1}\right)=\frac{1}{2}\left(\hat{p}_{1}+m\right)\left(1-P_{T} \gamma_{5} \hat{\eta}_{L}\right), \\
& u\left(k_{2}\right) \bar{u}\left(k_{2}\right)=\Lambda\left(k_{2}\right)=\hat{k}_{2}+m, \quad u\left(p_{2}\right) \bar{u}\left(p_{2}\right)=\Lambda\left(p_{2}\right),
\end{aligned}
$$

the square of matrix elements reads:

$$
\begin{aligned}
\left|M_{R}\right|^{2}= & -M_{R}^{\alpha} \bar{M}_{R}^{\alpha}= \\
= & -\frac{1}{t_{1}^{2}} S\left(\Gamma_{\mu \alpha}\left(k_{2}, k_{1}\right), k_{1}, \bar{\Gamma}_{\nu \alpha}\left(k_{1}, k_{2}\right), k_{2}\right) S\left(\gamma_{\mu}, p_{1}, \gamma_{\nu}, p_{2}\right)- \\
& -\frac{1}{t_{1} t} S\left(\Gamma_{\mu \alpha}\left(k_{2}, k_{1}\right), k_{1}, \gamma_{\nu}, k_{2}\right) S\left(\gamma_{\mu}, p_{1}, \bar{\Gamma}_{\nu \alpha}\left(p_{1}, p_{2}\right), p_{2}\right)- \\
& -\frac{1}{t_{1} t} S\left(\gamma_{\mu}, k_{1}, \bar{\Gamma}_{\nu \alpha}\left(k_{1}, k_{2}\right), k_{2}\right) S\left(\Gamma_{\mu \alpha}\left(p_{2}, p_{1}\right), p_{1}, \gamma_{\nu}, p_{2}\right)- \\
& -\frac{1}{t^{2}} S\left(\gamma_{\mu}, k_{1}, \gamma_{\nu}, k_{2}\right) S\left(\Gamma_{\mu \alpha}\left(p_{2}, p_{1}\right), p_{1}, \bar{\Gamma}_{\nu \alpha}\left(p_{1}, p_{2}\right), p_{2}\right)+ \\
& +\frac{1}{t_{1} u} S\left(\Gamma_{\mu \alpha}\left(k_{2}, k_{1}\right), k_{1}, \bar{\Gamma}_{\nu \alpha}\left(k_{1}, p_{2}\right), p_{2}, \gamma_{\mu}, p_{1}, \gamma_{\nu}, k_{2}\right)+ \\
& +\frac{1}{t_{1} u} S\left(\Gamma_{\mu \alpha}\left(p_{2}, k_{1}\right), k_{1}, \bar{\Gamma}_{\nu \alpha}\left(k_{1}, k_{2}\right), k_{2}, \gamma_{\mu}, p_{1}, \gamma_{\nu}, p_{2}\right)+ \\
& +\frac{1}{t_{1} z_{2}} S\left(\Gamma_{\mu \alpha}\left(k_{2}, k_{1}\right), k_{1}, \gamma_{\nu}, p_{2}, \gamma_{\mu}, p_{1}, \bar{\Gamma}_{\nu \alpha}\left(p_{1}, k_{2}\right), k_{2}\right)+ \\
& +\frac{1}{t_{1} z_{2}} S\left(\gamma_{\mu}, k_{1}, \bar{\Gamma}_{\nu \alpha}\left(k_{1}, k_{2}\right), k_{2}, \Gamma_{\mu \alpha}\left(k_{2}, p_{1}\right), p_{1}, \gamma_{\nu}, p_{2}\right)+ \\
& +\frac{1}{t u} S\left(\gamma_{\mu}, k_{1}, \bar{\Gamma}_{\nu \alpha}\left(k_{1}, p_{2}\right), p_{2}, \Gamma_{\mu \alpha}\left(p_{2}, p_{1}\right), p_{1}, \gamma_{\nu}, k_{2}\right)+ \\
& +\frac{1}{t u} S\left(\Gamma_{\mu \alpha}\left(p_{2}, k_{1}\right), k_{1}, \gamma_{\nu}, k_{2}, \gamma_{\mu}, p_{1}, \bar{\Gamma}_{\nu \alpha}\left(p_{1}, p_{2}\right), p_{2}\right)+ \\
& +\frac{1}{t z_{2}} S\left(\gamma_{\mu}, k_{1}, \gamma_{\nu}, p_{2}, \Gamma_{\mu \alpha}\left(p_{2}, p_{1}\right), p_{1}, \bar{\Gamma}_{\nu \alpha}\left(p_{1}, k_{2}\right), k_{2}\right)+ \\
& +\frac{1}{t_{1} z_{2}} S\left(\gamma_{\mu}, k_{1}, \gamma_{\nu}, k_{2}, \Gamma_{\mu \alpha}\left(k_{2}, p_{1}\right), p_{1}, \bar{\Gamma}_{\nu \alpha}\left(p_{1}, p_{2}\right), p_{2}\right)- \\
&
\end{aligned}
$$




$$
\begin{aligned}
& -\frac{1}{u^{2}} S\left(\Gamma_{\mu \alpha}\left(p_{2}, k_{1}\right), k_{1}, \bar{\Gamma}_{\nu \alpha}\left(k_{1}, p_{2}\right), p_{2}\right) S\left(\gamma_{\mu}, p_{1}, \gamma_{\nu}, k_{2}\right)- \\
& -\frac{1}{u z_{2}} S\left(\Gamma_{\mu \alpha}\left(p_{2}, k_{1}\right), k_{1}, \gamma_{\nu}, p_{2}\right) S\left(\gamma_{\mu}, p_{1}, \bar{\Gamma}_{\nu \alpha}\left(p_{1}, k_{2}\right), k_{2}\right)- \\
& -\frac{1}{u z_{2}} S\left(\gamma_{\mu}, k_{1}, \bar{\Gamma}_{\nu \alpha}\left(k_{1}, p_{2}\right), p_{2}\right) S\left(\Gamma_{\mu \alpha}\left(k_{2}, p_{1}\right), p_{1}, \gamma_{\nu}, k_{2}\right)- \\
& -\frac{1}{z_{2}^{2}} S\left(\gamma_{\mu}, k_{1}, \gamma_{\nu}, p_{2}\right) S\left(\Gamma_{\mu \alpha}\left(k_{2}, p_{1}\right), p_{1}, \bar{\Gamma}_{\nu \alpha}\left(p_{1}, k_{2}\right), k_{2}\right) .
\end{aligned}
$$

\section{Appendix D Test output}

Here we present the results of the test as test.dat output file corresponding to:

\begin{tabular}{|c|c|c|c|c|}
\hline \multicolumn{5}{|c|}{$\begin{array}{l}\text { itest }=1 \\
\mathrm{v} \text { generation } \\
\text { rgen is generated probability } \\
\text { rcalc is calculated probability }\end{array}$} \\
\hline \multicolumn{5}{|c|}{$\begin{array}{ll}\text { Ebeam }=45.0 & \mathrm{GeV} \\
\end{array}$} \\
\hline \multicolumn{5}{|c|}{ theta $=90.0$ degrees in $\mathrm{CM}$ system } \\
\hline on times target polariza & $\begin{array}{l}t=1.00 \\
\text { of bins } 2\end{array}$ & m polar & on times & target polariza \\
\hline \multicolumn{5}{|c|}{$\begin{array}{l}\text { number of radiative events } 100000000 \\
\text { initial random number } 12\end{array}$} \\
\hline bin & $\mathrm{v}$ & rgen & rcalc & rgen/rcalc \\
\hline 1 & $0.6998 \mathrm{E}-03$ & 376.4 & 375.0 & 1.004 \\
\hline 2 & $0.1268 \mathrm{E}-02$ & 205.8 & 205.4 & 1.002 \\
\hline 3 & $0.1826 \mathrm{E}-02$ & 143.4 & 143.2 & 1.001 \\
\hline 4 & $0.2382 \mathrm{E}-02$ & 111.3 & 111.2 & 1.002 \\
\hline 5 & $0.2936 \mathrm{E}-02$ & 92.07 & 91.91 & 1.002 \\
\hline 6 & $0.3490 \mathrm{E}-02$ & 79.40 & 79.29 & 1.001 \\
\hline 7 & $0.4043 \mathrm{E}-02$ & 70.70 & 70.59 & 1.002 \\
\hline 8 & $0.4596 \mathrm{E}-02$ & 64.45 & 64.41 & 1.001 \\
\hline 9 & $0.5148 \mathrm{E}-02$ & 60.02 & 59.96 & 1.001 \\
\hline 10 & $0.5701 \mathrm{E}-02$ & 56.81 & 56.78 & 1.001 \\
\hline 11 & $0.6254 \mathrm{E}-02$ & 54.52 & 54.57 & 0.9991 \\
\hline 12 & $0.6806 \mathrm{E}-02$ & 53.26 & 53.15 & 1.002 \\
\hline 13 & $0.7358 \mathrm{E}-02$ & 52.40 & 52.38 & 1.000 \\
\hline 14 & $0.7910 \mathrm{E}-02$ & 52.27 & 52.19 & 1.001 \\
\hline 15 & $0.8463 \mathrm{E}-02$ & 52.69 & 52.54 & 1.003 \\
\hline
\end{tabular}

1) itest $:=1$ - the generation of $\rho(v)$ distribution and comparison it with the analytical cross section corresponding to the first formula in (18) (here and below all of invariants $v, t_{1}, z$ are in $\mathrm{GeV}^{2}$ ) 


$\begin{array}{rrrrr}16 & 0.9015 \mathrm{E}-02 & 53.38 & 53.40 & 0.9996 \\ 17 & 0.9567 \mathrm{E}-02 & 54.94 & 54.78 & 1.003 \\ 18 & 0.1012 \mathrm{E}-01 & 56.53 & 56.69 & 0.9973 \\ 19 & 0.1067 \mathrm{E}-01 & 59.35 & 59.17 & 1.003 \\ 20 & 0.1122 \mathrm{E}-01 & 62.15 & 62.29 & 0.9978\end{array}$

2) itest $:=2$ - the generation of $\rho\left(t_{1}\right)$ distribution and comparison it with the analytical cross section corresponding to the second formula in (18)

\begin{tabular}{|c|c|c|c|c|}
\hline \multicolumn{5}{|c|}{$\begin{array}{l}\text { t1 generation } \\
\text { rgen is generated probability } \\
\text { rcalc is calculated probability }\end{array}$} \\
\hline \multicolumn{5}{|c|}{ Ebeam $=45.0 \quad \mathrm{GeV}$} \\
\hline \multicolumn{5}{|c|}{ theta $=90.0$ degrees in $\mathrm{CM}$ system } \\
\hline \multicolumn{5}{|c|}{$\mathrm{P}=\mathrm{pb} * \mathrm{pt}=1.00 \quad$ beam polarization times } \\
\hline $\begin{array}{l}\text { numb } \\
\text { init } \\
\mathrm{v}=\end{array}$ & $\begin{array}{l}\text { er of radiati } \\
\text { ial random nu } \\
0.5749 \mathrm{E}-02\end{array}$ & $\begin{array}{l}\text { events } \\
\text { er } 12\end{array}$ & 0000000 & \\
\hline bin & t1 & rgen & rcalc & rgen/rcalc \\
\hline 1 & $-0.2852 E-01$ & 235.5 & 201.1 & 1.171 \\
\hline 2 & $-0.2638 E-01$ & 183.0 & 313.5 & 0.5836 \\
\hline 3 & $-0.2533 E-01$ & 30.60 & 28.38 & 1.078 \\
\hline 4 & $-0.2377 \mathrm{E}-01$ & 11.23 & 10.86 & 1.034 \\
\hline 5 & $-0.2228 E-01$ & 8.905 & 8.711 & 1.022 \\
\hline 6 & $-0.2081 E-01$ & 9.842 & 9.674 & 1.017 \\
\hline 7 & $-0.1933 E-01$ & 14.89 & 14.58 & 1.021 \\
\hline 8 & $-0.1763 \mathrm{E}-01$ & 76.89 & 73.02 & 1.053 \\
\hline 9 & $-0.1689 E-01$ & 73.09 & 74.37 & 0.9827 \\
\hline 10 & $-0.1518 \mathrm{E}-01$ & 11.62 & 11.45 & 1.015 \\
\hline 11 & $-0.1369 \mathrm{E}-01$ & 6.749 & 6.655 & 1.014 \\
\hline 12 & $-0.1212 \mathrm{E}-01$ & 7.542 & 6.696 & 1.126 \\
\hline 13 & $-0.1095 \mathrm{E}-01$ & 5.605 & 5.257 & 1.066 \\
\hline 14 & $-0.9398 E-02$ & 2.392 & 2.340 & 1.022 \\
\hline 15 & $-0.7939 E-02$ & 1.711 & 1.700 & 1.007 \\
\hline 16 & $-0.6487 \mathrm{E}-02$ & 1.385 & 1.381 & 1.003 \\
\hline 17 & $-0.5041 E-02$ & 1.206 & 1.205 & 1.001 \\
\hline 18 & $-0.3594 E-02$ & 1.142 & 1.132 & 1.009 \\
\hline 19 & $-0.2129 \mathrm{E}-02$ & 1.250 & 1.213 & 1.030 \\
\hline 20 & $-0.1505 E-03$ & 11.30 & 7.410 & 1.525 \\
\hline
\end{tabular}

3) itest $:=3$ - the generation of $\rho(z)$ distribution and comparison it with the analytical cross section corresponding to the third formula in (18) 


itest=3
z generation
rgen is generated probability
rcalc is calculated probability
Ebeam=45.0 GeV
theta=90.0 degrees in CM system
P=pb*pt=1.00 beam polarization times target polarization
number of bins 20
number of radiative events 100000000
initial random number 12
v= $0.5749 \mathrm{E}-02$
t1=-0.1437E-01

4) itest $:=4$ - the cross-check of the accuracy of the vector reconstruction for 5 random radiative events

itest $=4$

variable reconstruction

Ebeam $=45.0 \quad \mathrm{GeV}$

theta $=90.0$ degrees in CM system

$\mathrm{P}=\mathrm{pb} * \mathrm{pt}=1.00$ beam polarization times target polarization number of radiative events 5

initial random number 12 


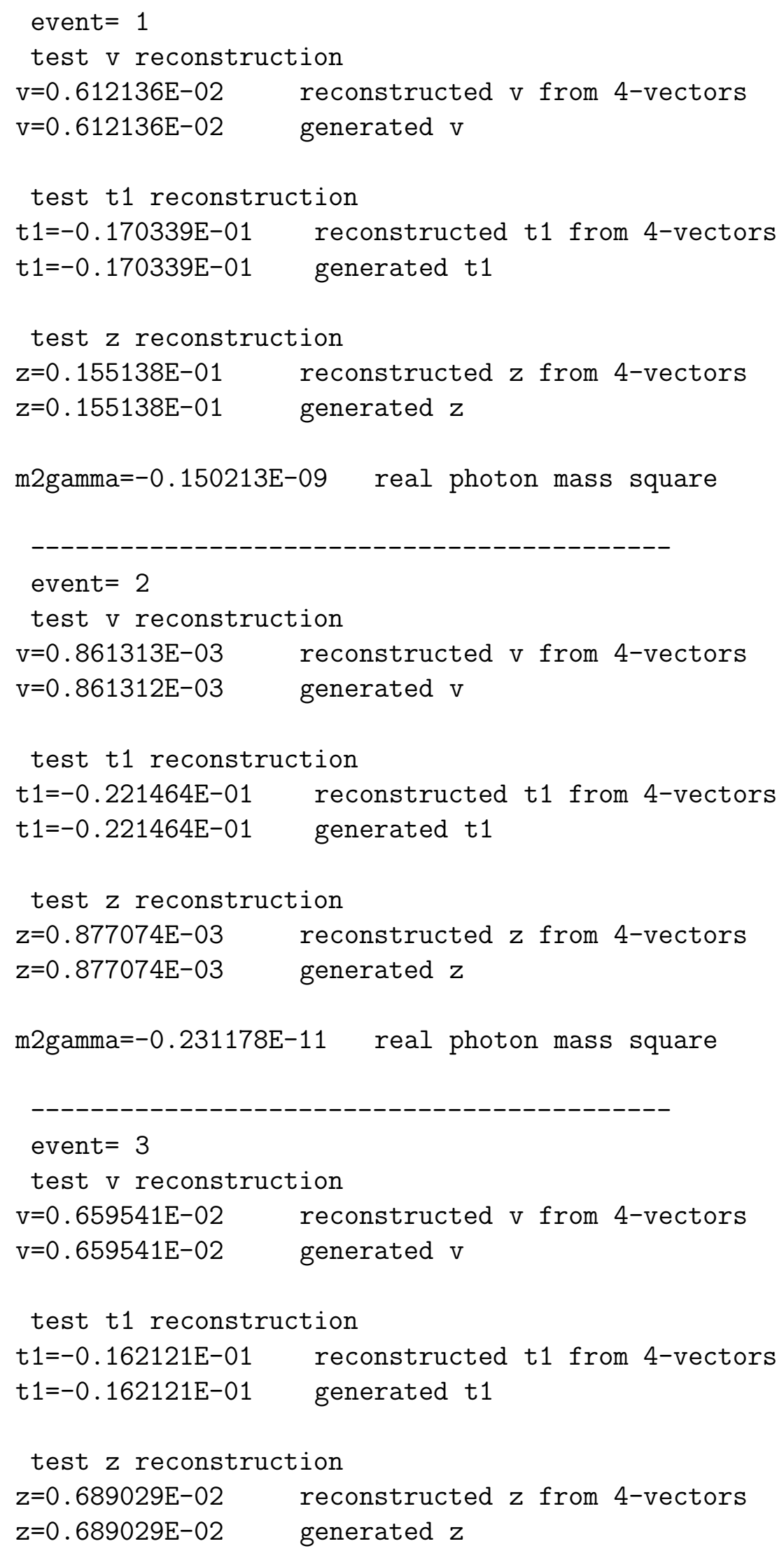


$\mathrm{m} 2$ gamma $=-0.710911 \mathrm{E}-10$ real photon mass square

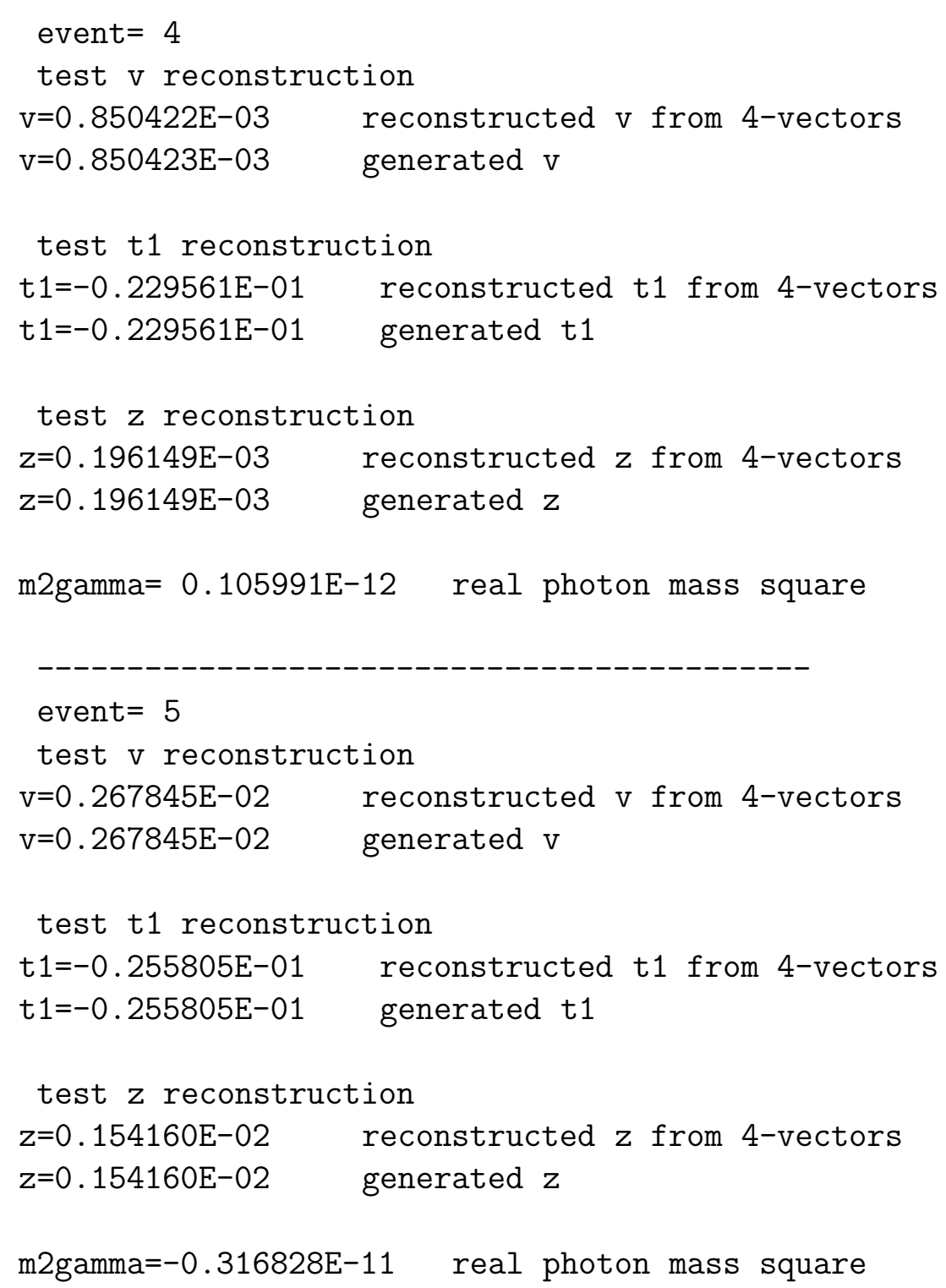

\section{References}

[1] M. Hauger et al., Nucl. Instrum. Meth. A 462 (2001) 382

[2] N.M. Shumeiko and J.G. Suarez J. Phys. G 26 (2000) 113

[3] A. Ilyichev and V. Zykunov, Phys. Rev. D 72 (2005) 033018

[4] Akushevich I., Boettcher H., Ryckbosch D., Proceedings Workshop "Monte Carlo Generators for HERA Physics" (1998/99). - Hamburg: DESY (1999) 554 
[5] Afanasev A., Akushevich I., Ilyichev A., Niczyporuk B., Czech. J. Phys. 53 (2003) 449

[6] V.A.Zykunov, Yad. Fiz. 67 (2004) 1366

[7] M.Böhm et al., Fortschr. Phys. 34 (1986) 687

[8] H.Burkhard and B.Pietrzyk, Phys. Lett. B356 (1995) 398 